\title{
Evidence of collision-induced resuspension of microscopic particles from a monolayer deposit
}

\author{
Amir Banari, ${ }^{1,}$ | Christophe Henry, ${ }^{2, \text { | }}$ Rafael Henrique Fank Eidt, ${ }^{1}$ \\ Pierre Lorenz, ${ }^{3}$ Klaus Zimmer, ${ }^{3}$ Uwe Hampel, ${ }^{1,4}$ and Gregory Lecrivain ${ }^{1,}$ 平 \\ ${ }^{1}$ Helmholtz-Zentrum Dresden-Rossendorf, Institut für Fluiddynamik, Bautzner Landstraße 400, 01328 Dresden, Germany \\ ${ }^{2}$ Université Côte d'Azur, Inria, CNRS, Cemef, France \\ ${ }^{3}$ Leibniz-Institut für Oberflächenmodifizierung, Permoserstraße 15, 04318 Leipzig, Germany \\ ${ }^{4}$ Technische Universität Dresden, Institut für Energietechnik, Professur für Bildgebende \\ Messverfahren für die Energie- und Verfahrenstechnik, 01069 Dresden, Germany
}

\begin{abstract}
The present letter addresses the resuspension of microscopic glass particles from a monolayer bed into a turbulent gas flow. With an intermediate surface coverage, here set to about $10 \%$ of the field of view, we report two distinct detachment mechanisms. At relatively low flow velocities, few loosely adhering particles move on the wall to eventually collide with neighboring particles resulting in a clustered resuspension. At higher fluid velocities, mostly individual particles resuspend due to their interaction with the turbulent flow. The resuspension curve, showing the remaining particle fraction as a function of the flow velocity, exhibits a strong bimodal character, that has not been reported so far.
\end{abstract}

Resuspension refers here to the physical process by which a bed of solid microscopic particles adhering to a surface is re-entrained into a gas due to the action of the flow. In the last decades, resuspension has received great interest owing to its importance in many environmental and industrial applications. Among the numerous examples that brings the significance of this topic, one can cite pollutant outbreak caused by strong winds [1], radioactive dust escaping nuclear reactors [2] or exposure to resuspendable airborne pathogens in indoor environments [3]. Despite a century of research, the underlying physical mechanisms behind particle resuspension in turbulent gas flows are still incomplete.

Shields 4] pioneered the field by reporting the existence of a threshold friction velocity beyond which resuspension occurs. Multiple empirical studies have since been performed and various estimates of this threshold friction velocity are now available [5, 6]. Since then, a number of experimental and numerical studies have investigated particle resuspension, usually making a distinction between monolayer and multilayer deposits.

In monolayer deposits, where particles tend to resuspend individually, previous experimental and theoretical studies have highlighted the mechanisms at play. Cleaver and Yates [7 made a first breakthrough by revealing the key role played by episodic turbulent bursts, similar in behavior to miniature tornadoes. They proposed a force-balance model to compute the resuspension rate of a monolayer bed of particles exposed to a turbulent boundary layer flow and suggested the direct particle liftoff as sole detachment mechanism. More recently, particles have been shown to exhibit three types of motion on the surface [8, 9, namely direct lift-off [10, 11], sliding

\footnotetext{
*a.banari@hzdr.de

$\dagger$ christophe.henry@inria.fr

$\ddagger$ g.lecrivain@hzdr.de
}

and rolling 12. Later improvements of the force-balance model followed by taking into account the statistical distribution of adhesive surface forces [13] and the balance between adhesion and aerodynamic torques [14]. Reeks and Hall [15] proposed an energy-balance model, where resuspension resulted from an accumulation of the fluctuating kinetic energy from the gas flow. Particles detached from the wall once the absorbed kinetic energy exceeded its adhesive counterpart. Such models were later refined to include wall roughness [16]. Using atomic force microscopy, surface roughness measurements and contact mechanics, Brambilla et al. [17, 18, revisited the calculation of the adhesion and lift torques induced by the lever arm. Some of latest developments found in the literature indicates that the resuspension of an isolated particle from a surface occurs following a combination of three mechanisms: (i) a particle is first set in motion by the fluid action, (ii) it then translates on the rough substrate by either rolling, sliding, or a combination of both, and (iii) it finally detaches from the surface to re-enter the flow upon colliding with a surface asperity [19, 20].

In multilayer deposits, where particles sit on top of one another [21, resuspension has been shown to depend on the initial particle arrangement and the inter-particle cohesion forces [22]. Several experimental and numerical studies, especially those involving the impact of incident particles 23, 24, have confirmed that an additional mechanism is at play in the re-entrainment of particles into the flow, that is the inter-particle collision, hereafter referred to as collision. For monolayer deposits, it remains to be seen when and whether collision plays a role. It has never been an essential ingredient in the theoretical development, because collision has not yet been observed to trigger resuspension in monolayer deposits. Recent experimental studies indicate however a possible role played by the collisions. Matsusaka 25] investigated the resuspension of 3.1 and $4.3 \mu \mathrm{m}$ alumina particles in a turbulent channel flow using a high-speed microscope. 
The images showed that the particle motion was initiated with a high initial velocity in arbitrary directions, often different from the channel streamwise direction. The authors attributed this observation to the possible collisions between previously deposited particles and those resuspending. Collisions were also suggested as a possible triggering mechanism by Rondeau et al. [26]. Although not observed directly, their study underlined that the high resuspension rate observed for non-spherical tungsten particles could only be explained by accounting for the collisions.

To summarize, there exists on the one hand a large body of literature on individual resuspension from monolayer deposits, in which the interparticle distance $(L)$ is usually much greater than the particle diameter $(d)$, that is $L / d \gg 1$. On the other hand, inter-particle collisions and deposit morphology were investigated in a number of studies on multilayer deposits, where the interparticle distance approaches zero, that is $L / d \rightarrow 0$. The present work bridges the gap by addressing the very special case, where the distance $L$ and the diameter $d$ have the same magnitude order. Such investigations raise exciting questions because of the anticipated competition between the two triggering mechanisms: resuspension induced by the turbulent flow and collisions. To the best of the authors' knowledge, this letter provides first experimental evidence of the crucial role played by collisions between microscopic particles even in the case of a monolayer bed produced at intermediate particle concentration. Quantitative numerical simulations endorse these observations by including a simple collision component to the course of events leading to resuspension. We stress, that this work is primary an experimental investigation and hence the model does not aim at exactly reproducing the experimental findings. The model, despite its relative simplicity, is presented to further highlight the subtle role played by local clustered regions, where the ratio $L / d$ nears unity.

To investigate the role of collision and its propagation within a monolayer deposit, an experimental test has been carried out. Using optical techniques, we track the motion of microscopic particles initially deposited on a wall surface with high spatial resolution. The experimental setup consists of a wind channel with a length of $2 \mathrm{~m}$ and square cross section of $h \times h=1 \mathrm{~cm}^{2}$, where $h$ is the channel height. A gas flow free of any impurities is created using pressurized nitrogen. The measurement section consists in a removable circular substrate. The substrate is a precision thin round window (EKSMA Optics, Lithuania) with extremely low wall roughness in the nanorange. It is located $1.5 \mathrm{~m}$ downstream of the channel inlet, a distance large enough to achieve a fully developed turbulent flow in the test section [27. It is fair to assume, that the nitrogen is at nearly atmospheric pressure in the measurement section. In the following experiments performed at room temperature of about $15{ }^{\circ} \mathrm{C}$, the nitrogen kinematic viscosity and density are estimated as $\nu=14.815 \times 10^{-6} \mathrm{~m}^{2} \cdot \mathrm{s}^{-1}$ and $\rho=1.185 \mathrm{~kg} \cdot \mathrm{m}^{-3}$.
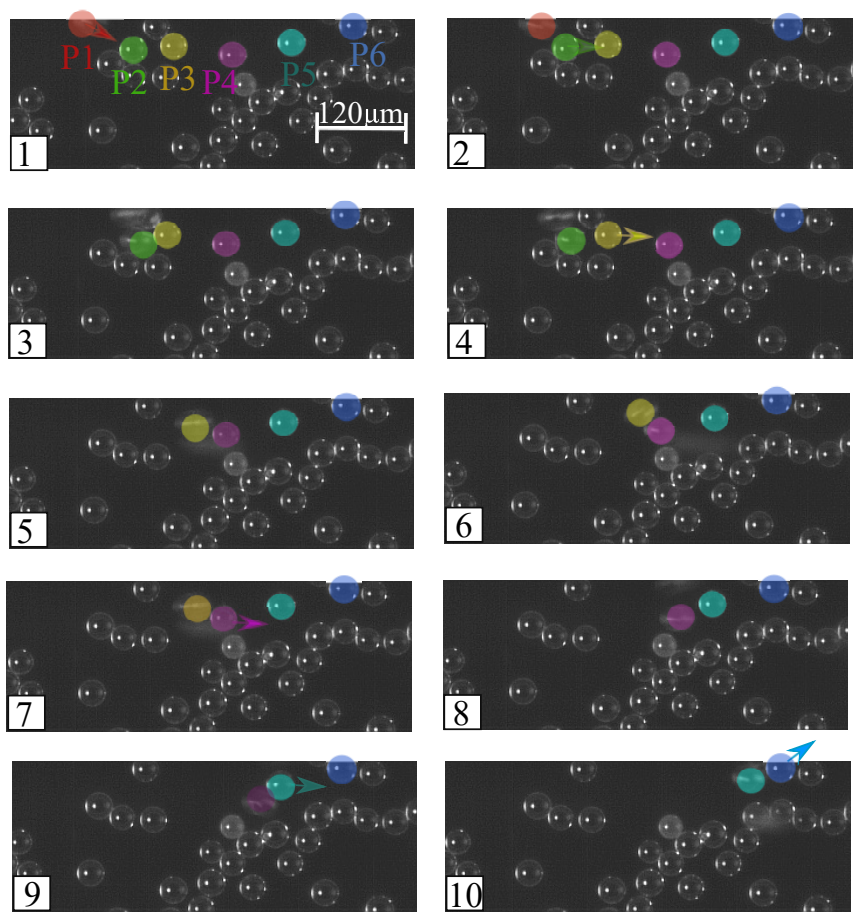

FIG. 1: Consecutive snapshots showing how collisions trigger a series of resuspension events. The time between two snapshots is $\Delta t=0.1 \mathrm{~ms}$, the flow rate is $Q=40 \mathrm{~L} / \mathrm{min}$ and the Reynolds number $R e=4500$.

Particles are initially placed manually on this substrate. The spherical particle beads used are made of Bariumtitanate glass ( $G B M 40$ from the Association of Powder Process Industry and Engineering), have a density of about $\rho_{p}=4200 \mathrm{~kg} \cdot \mathrm{m}^{-3}$ and are highly mono-disperse with a $40 \mu \mathrm{m}$ diameter. With a particle-to-gas density ratio $\rho_{p} / \rho \approx 3500$, the particles are much heavier than the ambient gas. Prior to experimenting, inner channel walls are cleaned and dried to ensure an oil and humidity free environment, that may adversely alter the resuspension. Each experiment starts with a monolayer deposit covering about $10 \%$ of the field of view. This corresponds to an average interparticle distance equaled to $L / d=2$. This coverage is generally greater than the one used in previous works on monolayer deposits [2, 15, and is purposely chosen to achieve a relatively high collision probability. We also ensured that all deposits remained monolayer. To monitor the number of particles $(N)$ on the surface, a high speed camera equipped with microscope lens is mounted above the substrate. The high-speed camera takes up to 10000 frames per second. Side and top LED lights brightens the measurement section for a better image processing. The frame recording starts at no flow rate and subsequently, the flow rate is increased in a stepwise fashion until $Q=300 \mathrm{~L} / \mathrm{min}$. This maximum flow rate corresponds to a Reynolds number $R e=U h / \nu$ $=33750$ with $U$ the corresponding mean flow velocity. Fig. 1 illustrates the snapshots of particles positions at 
10 consecutive time steps at a relatively low flow rate $Q=40 \mathrm{~L} / \mathrm{min}$ (i.e. $R e=4500$ ). Evidence of propagating collisions within the monolayer deposit is seen in this figure. The corresponding video is available in the supplementary material. In this temporal sequence of close-up views, a clustered resuspension is initiated by a "loosely adhering particle" labeled P1. It is first set in motion by aerodynamic forces; it then collides with a neighboring particle labeled $P 2$; this collision leads to the resuspension of particle $P 1$ and brings particle $P \mathscr{2}$ in motion; consecutive collisions with neighboring particles P3-P6 cause an avalanche of particle detachments.

In a larger field of view whose size is now $1.5 \mathrm{~mm}^{2}$, the number of remaining particles $N(R e, t)$ under constant flow, that is with fixed $R e$, is monitored until the monolayer deposit reaches an equilibrium state, that is when $N(R e, t \rightarrow \infty)=N_{\infty}(R e)$ becomes constant. This equilibrium state is usually reached in matter of seconds after being exposed to a fixed flow rate. Previous studies have shown that resuspension takes place in two stages. A first and short initial stage lasting some milliseconds is responsible for up to $80 \%$ of the entire resuspended mass 28. In a second stage, the detachment rate decays exponentially until, after some seconds, it is no longer detectable experimentally. In all presented experiments, we ensured the detachment rate no longer changed with time, that is when all particles with sufficiently low adhesive forces had resuspended under fixed Reynolds number. The remaining particles experiencing higher adhesion could only be resuspended at higher Re. Figure 2 shows the equilibrium states of an initial deposit obtained after some seconds at $R e=3375$ and $R e=4500$. We have been purposely colored resuspendable particles that form initial "clustered particle regions" on the surface, i.e. particles in close proximity with an initial inter-particle distance is in the order

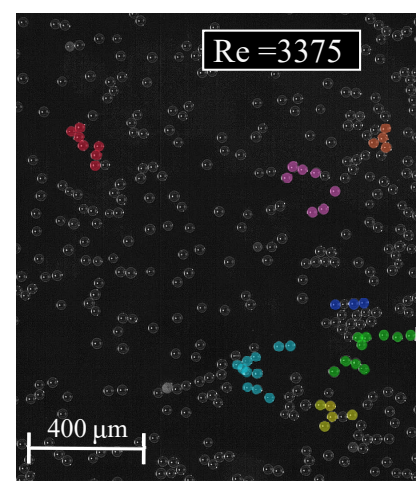

$\mathrm{RF}=100 \%$

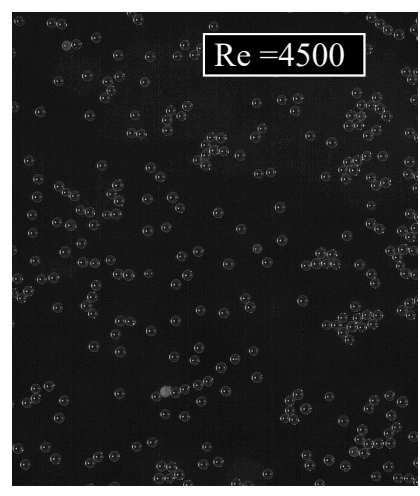

$\mathrm{RF}=82 \%$
FIG. 2: Equilibrium states of a monolayer deposit obtained after some seconds at $R e=3375$ and $R e=4500$. The re-entrained particles have been colored to highlight the local clustered regions where resuspension occurs. $R F$ is the remaining fraction. of some particle radii at most. Based on this definition, observations show that such clustered regions are usually formed by 4 to 20 particles. Once exposed to the gas flow, it can be seen that several resuspension events occur in localized regions where the local particle concentration is high. In that case, the resuspension of a single particle is followed by the collision with neighboring particles, leading to the resuspension of most of the particles in the clustered region. Besides, it appears that this collisioninduced regime occurs mostly at low velocities, typically $0<R e<5500$. In that case, $30 \%$ to $100 \%$ of the particles within the clustered region resuspend within a few increments in the Reynolds number.

With the help of suitable image processing algorithms, the remaining fraction $R F=N_{\infty}(R e) / N_{0}$ averaged over 11 repeated experiments is now reported, with $N_{0}$ being the particle number in the initial deposit. Figure 3 illustrates the evolution of $R F$ with increasing Reynolds number. A typical channel flow starts to transit from laminar to turbulent at critical Reynolds number $R e_{c}=2250$, that is here $Q=20 \mathrm{~L} / \mathrm{min}$. Fig. 3 shows that resuspension starts at $R e>R e_{c}$. This means that resuspension occurs here in the transitional and turbulent regimes. Surprisingly, the resuspension curve does not follow the typical sigmoid shape reported in previous investigations [2] but exhibits a strong bimodal character. Almost no resuspension occurs below $R e=2250$ at which point, the flow becomes turbulent. This means that resuspension occurs here in the transitional and turbulent regimes. This sudden observation of detachment is consistent with the work of Cleaver and Yates [7, who found a correlation between the turbulent coherent structures and the fluctuation of particle reentertainment. The sudden increase of particle detachment was explained due to existence of turbulent structures near the wall that interact with particles sticking to the wall.

Figure 3 shows a first descent with a steep gradient in the region $2500<R e<5500$, followed by a plateau, a second more pronounced descent in the region $13500<\operatorname{Re}<22500$, and a final exponential tail. This initial resuspension stage, where roughly $25 \%$ of the particles resuspend, is triggered by the motion of particles that loosely adhere to the wall surface. A loosely adhering particle is one, whose adhesion force is at the lower end of the adhesion force distribution [17]. More precisely, these loosely adhering particles start rolling and ram into their neighbors to eventually cause a local clustered resuspension. In larger clusters, multiple consecutive collisions were even found to unfold a resuspension avalanche. This means that the first steep gradient is associated with resuspension triggered by avalanches which prevail at low velocities and relatively high particle concentration. At higher velocities, typically for $R e>10000$, the average inter-particle distance increases because fewer particles remain on the surface and the largest initial clustered regions are gone. Experimental 


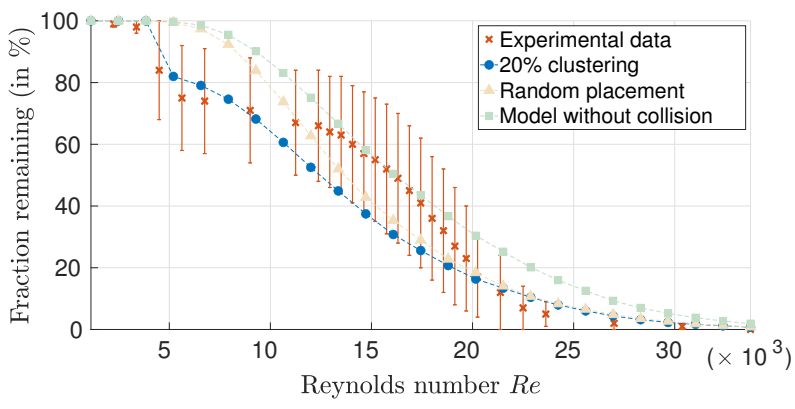

FIG. 3: Fraction of remaining particles (RF) with increasing $R e$. We discover a first descent in the region $2500<R e<5,500$ preceding a plateau.

observations showed that, at high $R e$, the bed is mostly composed of individual particles and some small clusters made of two to three particles at most, see figure 4 . As a result, both collision-propagation and usual individual resuspension events, driven by direct lift-offs or by rolling/sliding motion, occur. This means that, at high $R e$, if particles were initially separated by a large distance $L \gg d$, particle collisions would be rare and previously measured resuspension curves would be recovered. In reality, this picture is more complex since shielding effects also play a role [29. When particles are in close proximity, the downstream particles are shielded from the flow by those upstream, leading to more complex particle-flow interactions, which depend on the inter-particle distance and the near-wall turbulence within the deposit. This shielding effect is left out of the present paper since the fluid motion has not been measured experimentally.

To further highlight these clustered resuspension events, the experimental data are compared to numerical simulations obtained with a relatively simple extension

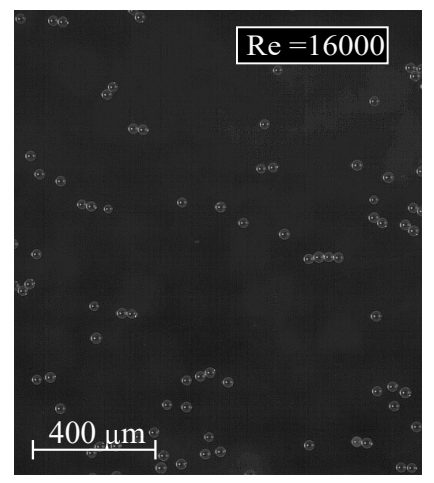

$\mathrm{RF}=20 \%$

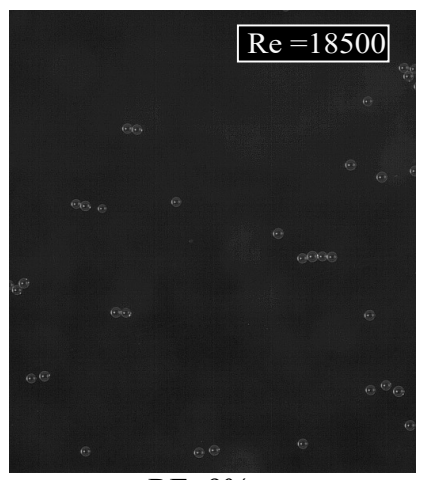

$\mathrm{RF}=9 \%$
FIG. 4: Equilibrium states at $R e=16000$ and $R e=18500$. At higher Reynolds number the bed is mostly composed of individual particles and some small clusters made of two to three particles at most. of an existing resuspension model, that was previously developed and validated by the authors [20, 30. This extension is based on a two-step scenario: first, particles are set into motion using a force/torque balance model and, second, the motion of each moving particle is used to determine if it collides with another deposited particle. The first step is computed using typical force-balance models 31 which describe the three resuspension mechanisms: direct lift-off, sliding or rolling. More precisely, a particle resuspends due to the rupture of the balance between torques/forces associated to aerodynamic drag $\left(F_{d}\right)$, lift $\left(F_{\ell}\right)$, adhesion $\left(F_{a d h}\right)$ forces, and gravity $(g)$ :

$$
\begin{array}{cc}
F_{\ell}>F_{a d h}+m_{p} g & \text { lift-off } \\
F_{d}>\mu_{s}\left(F_{a d h}+m_{p} g-F_{\ell}\right) & \text { sliding } \\
1.4 r_{p} F_{d}+a F_{\ell}>a\left(F_{a d h}+m_{p} g\right) & \text { rolling }
\end{array}
$$

with $m_{p}$ the particle mass, $r_{p}$ its radius, $\mu_{s}$ the static friction coefficient, and $a$ the length of the lever-arm, that is the distance from the particle center of mass to the downstream contact point where the particle pivots. This distance as well as the adhesion force are evaluated numerically by modeling the rough substrate as a smooth plate covered by hemispherical asperities. Drag and lift are evaluated by randomly generating the instantaneous fluid velocity at the particle position following existing near-wall correlations for the mean and fluctuating fluid velocity components. Further details are found in 20 . The factor 1.4 in the torque of drag forces accounts for the anisotropy of the fluid near the surface 32. To account for the collision propagation, the model has been extended as follows: when a particle starts moving in the first step, we evaluate the probability that this particle collides with another one still deposited on the surface. Following the first experimental observations, we further consider that, upon colliding with a deposited particle, the moving particle is immediately resuspended while the impacted particle starts moving. In turn, the impacted particle has a chance to collide with other deposited particles on the surface and so on. For each moving particle, the chances of collision with a deposited particle are evaluated using a geometric consideration. For the sake of simplicity, each resuspended particle is assumed to move with a velocity equal to the fluid velocity at the particle position, regardless of the type of motion. This fluid velocity is sampled for each particle using the empirical formulas from Matida et al. [33, who provide an estimation of the mean and fluctuating parts of the fluid velocity in the near-wall region. This allows to evaluate the distance traveled by each moving particle before it becomes separated by more than twice the particle radius, at which point a collision with another deposited particle becomes impossible. If a deposited particle is present within the path of the particle as it moves close to the surface, a collision propagation event is triggered.

Despite its relative simplicity, this collision model already provides comparison data that would else be unavailable. Numerical simulations are performed for a series of flow rates. In line with the experimental setup, we 


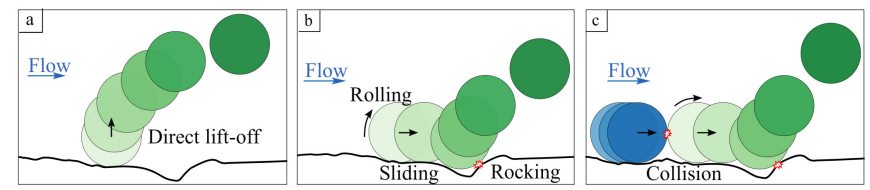

FIG. 5: Sketch of the five mechanisms (lift-off, sliding, rolling, rocking, collision) in monolayer resuspension.

monitor the number of particles remaining on the surface after an exposure of $1 \mathrm{~s}$ to a given flow rate, that is until a steady state is obtained. The simulations are set using experimental information on particle, fluid ans substrate properties. We set here the particle diameter to $40 \mu \mathrm{m}$, the particle density to $4.200 \mathrm{~kg} \cdot \mathrm{m}^{-3}$, a surface coverage to $10 \%$, the fluid properties to those obtained at $15{ }^{\circ} \mathrm{C}$, a wall roughness size to $\sim 100 \mathrm{~nm}$ and a Hamaker constant to $A_{\text {Ham }}=10^{-20} \mathrm{~J}$. Three different simulations have been performed: (i) the model with the first step only, that is without collision effects, and starting with particles placed randomly on the surface, (ii) the full model with randomly placed particles and (iii) the full model with $20 \%$ of the particles being placed in a cluster initially and the rest being randomly placed. The results obtained are displayed in Fig. 3. It is seen that the green curve obtained with setup (iii) is in relatively good agreement with the experimental data, including the steep decrease at low Reynolds number Re. Comparing the results to those obtained without initial particle clustering (setup ii, blue curve), it clearly appears that this steep decrease is associated to the presence of such clustered regions. This can be explained as follows: in high concentration regions, the chances of collision with another particle is much higher due to the lower interparticle distance compared to non-clustered regions. As a result, without clustering, the chance of collision is homogeneous across the whole region leading to a sigmoid shape that resembles the one obtained without the model with collisions (yellow curve). Comparing the setup (i) and (ii), it can be seen that collision propagation does lead to a slightly higher resuspension rate but that it does not account for the initial steep decrease. These results thus confirm the key role played by collision propagation associated with a non-homogeneous initial particle repartition on the surface.

To conclude, we have provided here direct experimental evidence showing the role of collisions in the resuspension process even for monolayer deposits with a surface coverage as low as $10 \%$. As summarized in Fig. 5 , these findings support the idea that, similarly to multilayer resuspension, monolayer resuspension does result from the three following mechanisms: (a) direct liftoff, (b) rolling/sliding motion followed by rocking events and (c) inter-particle collisions. These first findings also raise new questions about the exact collision propagation mechanism. It appears indeed that the outcome of such collisions depends strongly on inter-particle distances and thus clustering. Yet, the result of the collision should also depend on the kinetic energy of the impacting particle, its angle of impact with respect to the deposited particle as well as on the adhesion properties with the surface. More careful and systematic analysis of the experimental materials will be carried out in the near future to provide deeper insights into this mechanism, including shielding effect, as well as to develop more sophisticated models that include both inter-particle collisions, including for instance elastic and inelastic collisions, and fine models for the particle dynamics near the wall.

\section{ACKNOWLEDGMENTS}

This work was funded by the Helmholtz Associations Initiative and Networking Fund within the frame of the Helmholtz Climate Initiative (HI-CAM). The authors are responsible for the content of this publication.
[1] S. Brambilla, S. Speckart, and M. J. Brown, Adhesion and aerodynamic forces for the resuspension of nonspherical particles in outdoor environments, J. Aerosol Sci. 112, 52 (2017)

[2] T. Barth, J. Preuß, G. Müller, and U. Hampel, Single particle resuspension experiments in turbulent channel flows, J. Aerosol Sci. 71, 40 (2014).

[3] I. Rivas, J. C. Fussell, F. J. Kelly, and X. Querol, Indoor sources of air pollutants, in Indoor Air Pollution (The Royal Society of Chemistry, 2019) pp. 1-34.

[4] A. Shields, Application of similarity principles and turbulence research to bed-load movement, California Institute of Technology (1936).

[5] T. Barth, G. Lecrivain, S. Jayaraju, and U. Hampel, Particle deposition and resuspension in gas-cooled reactors- activity overview of the two european research projects thins and archer, Nucl. Eng. Des. 290, 127 (2015)

[6] W. Wu, G. Soligo, C. Marchioli, A. Soldati, and U. Piomelli, Particle resuspension by a periodically forced impinging jet, J. Fluid Mech. 820, 284 (2017).

[7] J. Cleaver and B. Yates, Mechanism of detachment of colloidal particles from a flat substrate in a turbulent flow, J. Colloid Interface Sci. 44, 464 (1973).

[8] E. Rabinovich and H. Kalman, Incipient motion of individual particles in horizontal particle-fluid systems: A. experimental analysis, Powder Technology 192, 318 (2009).

[9] E. Rabinovich and H. Kalman, Incipient motion of individual particles in horizontal particle-fluid systems: B. theoretical analysis, Powder Technology 192, 326 (2009) 
[10] R. Shnapp and A. Liberzon, A comparative study and a mechanistic picture of resuspension of large particles from rough and smooth surfaces in vortex-like fluid flows, Chemical Engineering Science 131, 129 (2015)

[11] H. Traugott and A. Liberzon, Experimental study of forces on freely moving spherical particles during resuspension into turbulent flow, International Journal of Multiphase Flow 88, 167 (2017)

[12] Y. Jiang, S. Matsusaka, H. Masuda, and Y. Qian, Characterizing the effect of substrate surface roughness on particle-wall interaction with the airflow method, Powder Technology 186, 199 (2008).

[13] H. Wen and G. Kasper, On the kinetics of particle reentrainment from surfaces, J. Aerosol Sci. 20, 483 (1989).

[14] H.-C. Wang, Effects of inceptive motion on particle detachment from surfaces, Aerosol Sci. Technol. 13, 386 (1990)

[15] M. Reeks, J. Reed, and D. Hall, On the resuspension of small particles by a turbulent flow, J. Phys. D: Appl. Phys. 21, 574 (1988).

[16] M. Reeks and D. Hall, Kinetic models for particle resuspension in turbulent flows: theory and measurement, J. Aerosol Sci. 32, 1 (2001)

[17] M. N. Rush, S. Brambilla, S. Speckart, G. A. Montaño, and M. J. Brown, Glass-particle adhesionforce-distribution on clean (laboratory) and contaminated (outdoor) surfaces, Journal of Aerosol Science 123, 231 (2018)

[18] S. Brambilla and M. J. Brown, Impact of the adhesionforce lever-arm "a" on the rock ' $\mathrm{n}$ ' roll resuspension model and how to compute it from contact mechanics, J. Aerosol Sci. 143, 105525 (2020)

[19] M. Guingo and J.-P. Minier, A new model for the simulation of particle resuspension by turbulent flows based on a stochastic description of wall roughness and adhesion forces, J. Aerosol Sci. 39, 957 (2008)

[20] C. Henry and J.-P. Minier, Colloidal particle resuspension: On the need for refined characterisation of surface roughness, J. Aerosol Sci. 118, 1 (2018)

[21] G. Lecrivain, A. Vitsas, A. G. Boudouvis, and U. Hampel, Simulation of multilayer particle resuspension in an obstructed channel flow, Powder Technology 263, 142 (2014).

[22] M. Lazaridis and Y. Drossinos, Multilayer resuspension of small identical particles by turbulent flow, Aerosol Sci. Technol. 28, 548 (1998).

[23] D. Beladjine, M. Ammi, L. Oger, and A. Valance, Collision process between an incident bead and a threedimensional granular packing, Phys. Rev. E 75, 061305 (2007)

[24] L. Oger, M. Ammi, A. Valance, and D. Beladjine, Study of the collision of one rapid sphere on 3d packings: Experimental and numerical results, Comput. Math. Appl. $\mathbf{5 5}, 132$ (2008).

[25] S. Matsusaka, High-resolution analysis of particle deposition and resuspension in turbulent channel flow, Aerosol Sci. Technol. 49, 739 (2015)

[26] A. Rondeau, S. Peillon, A. M. Vidales, J. Benito, R. Uñac, J.-C. Sabroux, and F. Gensdarmes, Evidence of inter-particles collision effect in airflow resuspension of poly-dispersed non-spherical tungsten particles in monolayer deposits, J. Aerosol Sci. 154, 105735 (2021).

[27] F. M. White, Fluid Mechanics (McGraw Hill, 2011).

[28] F. Zhang, M. Reeks, M. Kissane, and R. Perkins, Resuspension of small particles from multilayer deposits in turbulent boundary layers, Journal of Aerosol Science 66, $31(2013)$

[29] R. Beetstra, M. van der Hoef, and J. Kuipers, A latticeboltzmann simulation study of the drag coefficient of clusters of spheres, Computers \& Fluids 35, 966 (2006), proceedings of the First International Conference for Mesoscopic Methods in Engineering and Science.

[30] C. Henry and J.-P. Minier, Progress in particle resuspension from rough surfaces by turbulent flows, Prog. Energy Combust. Sci. 45, 1 (2014).

[31] A. Ibrahim, P. Dunn, and M. Qazi, Experiments and validation of a model for microparticle detachment from a surface by turbulent air flow, J. Aerosol Sci. 39, 645 (2008)

[32] M. O'neill, A sphere in contact with a plane wall in a slow linear shear flow, Chemical Engineering Science 23, 1293 (1968)

[33] E. A. Matida, K. Nishino, and K. Torii, Statistical simulation of particle deposition on the wall from turbulent dispersed pipe flow, International Journal of Heat and Fluid Flow 21, 389 (2000). 